\title{
Balanceo de líneas de producción en la industria farmacéutica mediante Programación por metas
}

\section{Production line balancing in the pharmaceutical industry using Goal Programming}

DOI: http://dx.doi.org/10.17981/ingecuc.15.1.2019.10

Artículo de Investigación Científica. Fecha de Recepción: 29/08/2018. Fecha de Aceptación: 16/02/2019.

\author{
Juan Pablo Orejuela Cabrera (iD \\ Universidad del Valle. Cali, (Colombia) \\ juan.orejuela@correounivalle.edu.co
}

\author{
Andrés Flórez González \\ Universidad del Valle. Cali, (Colombia) \\ andresflorezgonzalez@gmail.com
}

Para citar este artículo:

Juan Orejuela-Cabrera y Andrés Flórez-González, "Balanceo de líneas de producción en la industria farmacéutica mediante Programación por metas," INGE CUC, vol. 15, no. 1, pp. 109-122, 2019. DOI: http://doi.org/10.17981/ingecuc.15.1.2019.10

\begin{abstract}
Resumen
Introducción- En una línea de fabricación es muy importante que los tiempos de ciclo de las diferentes estaciones estén balanceados y que sean bajos, ya que esto permite disminuir los inventarios de producto en proceso, sin embargo, hacer esto conlleva a aumentar el número de estaciones, lo que no es favorable ya que eleva los costos fijos asociados a las estaciones, en tal sentido es necesario definir estrategias que permitan lograr un equilibrio entre estos requerimientos.
\end{abstract}

Objetivo- En este artículo se propone la formulación de un modelo para el balanceo de línea, utilizando la técnica de programación multi-objetivo por metas, aplicada a la industria farmacéutica con el fin de minimizar el número de estaciones, minimizar el tiempo de ciclo y el inventario en proceso.

Metodología- Se emplea la programación por metas para abordar un modelo de balance de línea, que considera al mismo tiempo la asignación de múltiples estaciones una operación y la asignación de múltiples operaciones a una estación.

Resultados-Se logra una reducción significativa del tiempo ciclo y del tiempo ocioso a costos mínimos, además se presenta una comparación entre el modelo determinístico y estocástico.

Conclusiones- A través de esta implementación del modelo en LINGO, se validó el cumplimiento de las restricciones planteadas, la precedencia de las operaciones y el buen funcionamiento del modelo mediante las soluciones óptimas obtenidas. La simulación, es una herramienta que permite ilustrar la complejidad de las operaciones del sistema de producción, las cuales requieren como en nuestro caso de una modelación más ajustada a la realidad para comprender el comportamiento del proceso y evaluar diferentes estrategias.

Palabras clave- Balanceo de línea; Tiempo de ciclo; Programación por metas; Modelo Matemático; Enfoque Multiobjetivo.

\begin{abstract}
Introduction- In a production Line it's important that the stations' cycle times are balanced and that they are low since this allows to reduce the work in process. However, doing this leads to an increase in the stations' number, that is not favorable because it raises the costs associated with the stations, therefore it is necessary to define strategies that allow achieving a balance between these requirements.
\end{abstract}

Objective-In this article we propose the formulation of a model for the line balancing, using the technique of multi-objective goal programming, applied to the pharmaceutical industry in order to minimize the stations' number, minimize cycle time and inventory in process.

Methodology-Goal programming is used to address a line balance model, which considers at the same time the assignment of multiple stations to one operation and the assignment of multiple operations to one station.

Results- A significant decrease in cycle time and idle time at minimum costs is achieved, and a comparison between the deterministic and stochastic models is presented.

Conclusions- Through this implementation of the LINGO model, the compliance of the proposed restrictions, the precedence of operations and the proper functioning of the model were validated through the optimal solutions obtained. The simulation is a tool that illustrates the complexity of the operations of the production system, which require, as in our case, a more realistic modeling to understand the behavior of the process and evaluate different strategies.

Keywords- Line Balancing; Cycle time; Goal Programming mathematical model; Multi-Objective approach. 


\section{INTRODUCCIÓN}

El problema general de los sistemas de producción consiste en definir la correcta asignación de recurso a los procesos de fabricación, para lograr un equilibrio que ayude a encontrar la forma de minimizar costos, mantener la calidad y usar el tiempo eficientemente. El balanceo de línea, contribuye estos propósitos, ya que su fin es el de asegurar un flujo continuo y uniforme de los productos, acomodando las operaciones en estaciones de tal forma que se equilibren los tiempos.

Una línea de ensamble es un sistema productivo donde las operaciones se encuentran alineadas en estaciones alrededor de una pieza de trabajo. Estas piezas, visitan las estaciones a lo largo de la línea usando generalmente algún sistema de transporte automático [1].

Originalmente, las líneas de ensamble fueron diseñadas para obtener eficiencia de los costos en la producción en masa de artículos estandarizados, sin embargo, para responder a las diversas necesidades de los clientes, las compañías han buscado la individualización de sus artículos o lo que comúnmente se conoce como mass customization [1].

Las líneas de ensamble se emplean con mayor frecuencia en la fabricación de productos masivos estandarizados [2], en ambientes que por lo general trabajan contra inventario y que buscan maximizar la eficiencia del sistema. Inicialmente, las líneas de ensamble presentaban limitaciones en otros entornos más dinámicos, sin embargo, nuevas formas de balance de línea han surgido y estas permiten tener mucha más flexibilidad, lo cual facilita su uso en otros ambientes de fabricación.

Los diferentes problemas relacionados con el balanceo de línea, en función de los componentes que se consideren, las restricciones y funciones de desempeñó, se clasifican en dos grandes categorías [3]:

Problemas Simples del Balanceo de Línea $(S A L$ $B P)$, estos se subdividen según los objetivos considerados, el $S A L B P$-1 que dado un tiempo de ciclo minimiza el número de estaciones, el $S A L B-2$ que dado el número de estaciones minimiza el tiempo de ciclo.

Problemas Generales del Balanceo de Línea $(G A L$ $B P)$. Este último abarca todos los problemas que no alcanza a cubrir el $S A L B P$ como, por ejemplo: los problemas multi-objetivo, las estaciones en paralelo, líneas en forma de U, tiempo de procesos variables, entre otros.

En las últimas décadas, varios investigadores se han preocupado por el efecto de los almacenamientos entre estaciones de trabajo en una línea productiva ya que la inversión en inventario de trabajo en proceso (WIP Inventory) y otros costos como los de almacenamiento y transporte repercuten en su desempeño. Sin embargo reducir el inventario WIP, no necesariamente aumenta el desempeño de una línea de ensamble y la meta de cero inventarios es casi inalcanzable [4].
La literatura especializada reporta diferentes estudios dedicados a determinar la eficiencia de una línea productiva y el efecto de la capacidad de sus almacenes intermedios. Se han desarrollado diversas expresiones exactas y métodos numéricos para líneas de un tamaño limitado. También, diversas aproximaciones y técnicas de simulación han sido desarrolladas para las líneas más extensas.

En [5] se examina el efecto de la transferencia de un lote discreto de inventario WIP, considerando cero el tiempo de transporte, utilizando un modelo de simulación. La transferencia del inventario WIP involucra la determinación del número de contenedores a ubicar en cada estación y sus capacidades.

Complementariamente, en [6] se estudia el problema del control de flujo de material con vehículos guiados automáticamente ( $A G V$, por sus siglas en ingles), donde los productos se mantienen en ellos mientras están siendo procesados, esto debido a la flexibilidad de los $A G V$ introducida en este modelo. Aquí, se desarrollan modelos para la gestión y el control del flujo de material, donde su uso permite al diseñador determinar el tiempo óptimo de lanzamiento a la línea de cargas sucesivas para minimizar el makespan (tiempo de terminación del último ítem), mientras se balancean los tiempos ociosos de la línea y de los $A G V$.

Generalmente, para facilitar el movimiento entre estaciones y el procesamiento simultaneo en distintas máquinas, se utilizan lotes de cargas, lo cual aumenta la utilización de los recursos y el tiempo de terminación del lote. En [7] se hace referencia al problema de producir cargas unitarias de un mismo tamaño en múltiples etapas con consideraciones de manejo de material, donde por medio de un algoritmo heurístico basado en programación dinámica se busca minimizar el tiempo de terminación del lote.

Los tamaños de lote, pueden variar desde diez piezas hasta miles y a menos que se esté ejecutando un adecuado control, se pueden ver las fuentes potenciales de mejoramientos. Las dos principales actividades son las operaciones en las estaciones y los traslados de materiales.

En [8], se diseña una metodología para seleccionar los parámetros de las máquinas en cada estación y un plan de manejo del material WIP, en un sistema de producción multi-etápico, donde el principal objetivo es disminuir el costo total de producción.

Adicionalmente, en [9] se presenta una posible solución práctica para un sistema de control del WIP manejable y bien distribuido. Se tienen en cuenta los avances en las tecnologías computacionales para el control del WIP para que puedan ser generados desde un servidor. Estas instancias del control del WIP son coordinadas y sincronizadas usando el concepto de líneas de producción virtuales $(V P L)$. Se proponen algoritmos para el control del WIP, para implementar operaciones "Pull" en tiempo real. 
El sistema de suministro de partes en un taller de ensamble asume la función de suministrar las partes de ensamble, desde una bodega hasta las estaciones de trabajo del taller de ensamble. Este sistema juega un papel estratégico en la determinación del nivel de inventario en una línea de ensamble. En [10] se propone un sistema de suministro de partes dinámico para una planta de ensamble de automotores la cual estima las cantidades de consumo dinámicamente, considerando el progreso actual de la producción. Los resultados de una simulación muestran que el sistema propuesto produce mejores resultados, en comparación con los que produce un sistema estático de suministro de partes.

En la presenteinvestigación, sebusca la disminución del inventario, en una línea de ensamble en la que hay operaciones con un largo tiempo de proceso, que dificulta el balance en un contexto tradicional, para ello se propone un modelo para el balanceo de línea, utilizando la técnica de programación multi-objetivo por metas en la industria farmacéutica.

El modelo, pretende minimizar el tiempo de ciclo de la forma usual, y se plantea como principal aporte del trabajo la inclusión de la posibilidad de que una operación, cuyo tiempo de proceso sea muy largo, se lleve a cabo simultáneamente en varias estaciones, distribuyendo así el tiempo de la operación entre las estaciones agrupadas, lo que permite balancear la línea y disminuir el tiempo de ciclo total de la línea y por ende el inventario de producto en proceso.

Los planteamientos anteriores, implican que el modelo permite al mismo tiempo asignar varias operaciones de tiempo de proceso corto a una misma estación y asignarle a una operación de tiempo de proceso largo varias estaciones; lo cual genera la posibilidad de contar con dos formas diferentes de cálculo de tiempo de ciclo para las estaciones, representado a su vez, un desafío importante en la formulación del problema.

Además, se propone un modelo de programación por metas, en el que las metas son no preferentes y se construye una función objetivo que busca la minimización de los costos asociados: al tiempo de ciclo, al número de estaciones y a la agrupación de éstas para una operación, estableciendo unas metas relacionadas con las estaciones totales a utilizar y el número máximo de estaciones que se pueden agrupar por operación.

En otras palabras, el problema de balance de línea que se aborda en esta investigación es un $G A L B P$, que busca minimizar, al mismo tiempo, el número de estaciones, el tiempo de ciclo y las penalizaciones asociadas a asignar más estaciones de las planeadas y más operaciones de las planeadas a una estación. Además, considera la posibilidad de asignar una actividad más de una estación.

El manuscrito se ha organizado con la siguiente estructura, en la sesión II se presenta una revisión de la literatura sobre los referentes teóricos y empí- ricos asociados al balance de línea y sus técnicas de solución, en la sección III se presenta el desarrollo metodológico para el problema planteado, en la sección IV se presentan los resultados de aplicación del modelo en una industria farmacéutica, y finalmente, en la sección $\mathrm{V}$ se presentan las conclusiones.

\section{Referentes Teóricos y Empíricos}

En las últimas tres décadas, nuevas metodologías han sido desarrolladas con el propósito de obtener mejores soluciones; por ejemplo, [11] desarrollan el procedimiento de ramificar y acotar para el Problema Simple de Balanceo de Línea tipo 2 o problema de asignar tareas a un número determinado de estaciones de trabajo con el fin de maximizar la tasa de producción.

Más tarde, [12] se basa en la técnica de las hormigas con el fin de tratar efectivamente el Problema de Balanceo de Línea tipo 1, el objetivo es minimizar el número de estaciones de trabajo dado un tiempo de ciclo.

De otra parte, en la literatura especializada, se reportan numerosos trabajos que tratan este tipo de problema, de lo que [13] presentan un estado del arte totalmente actualizado de los procedimientos exactos y heurísticos para resolver $S A L B P$; en dicho trabajo, los autores concluyen que, los métodos disponibles que resuelven instancias de problemas de tamaño medio son efectivos para ser usados en la práctica, pero que requieren mejoras para que puedan resolver problemas de mayor escala.

En [14]the constraint programming (CP, se da un enfoque de programación de restricciones $(C P)$ se aplica para problema simple de balance de la línea de ensamblaje ( $S A L B P$ ), así como algunas de sus generalizaciones. $C P$ es un lenguaje de modelado enriquecido que permite formulación de problemas combinatorios generales y se combina con un sólido conjunto de métodos de solución.

En [15], se estudia un problema de balance de línea de ensamblaje con incertidumbre en los tiempos de cada tarea. A fin de que lidiar con la incertidumbre, se propone una formulación sólida para manejar los cambios en los tiempos de operación.

En [16], se aborda el problema balance de línea en U teniendo en cuenta los riegos económicos, para ello consideran la manipulación de carga, la repetitividad de actividades y las posturas forzadas o incomodas. Los autores presentan diferentes métodos ergonómicos y evidencian el balance de línea en las estaciones de trabajo bajo un nivel aceptable ergonómico.

Paralelo a este desarrollo en los problemas de balanceo de línea también se dieron avances en la rama de la programación matemática multi-objetivo. [17] introduce la programación lineal como recurso para dar solución a los problemas de balanceo de línea mono objetivo en su trabajo; luego, [18] expone el marco teórico de la programación matemática multi-objetivo sin presentarlo en un caso específico. 
Por su parte, en [19] se presenta un modelo de programación matemática que muestra de una manera explícita una función con más de un objetivo a optimizar; tal como sucede en la mayor parte de los casos resulta imposible satisfacer exactamente todos los objetivos. La función objetivo del programa, consiste en la minimización de la suma de los valores absolutos de las desviaciones (positivas o negativas) producidas para cada objetivo, con respecto a sus niveles de logro establecidos a priori. Esta técnica ha sido denominada: programación multi-objetivo por metas.

La programación por objetivos experimenta un fuerte impulso con la publicación del libro de Ijiri [20]; este trabajo presenta de una manera perfeccionada, tanto el marco teórico como la estructura operativa propuesta por [19]. La principal novedad teórica de [20], consiste en la introducción de las llamadas prioridades excluyentes, es decir, el logro de las metas que se encuentran situadas en un cierto nivel de prioridad son absolutamente preferidas a la realización de cualquier otro conjunto de metas situadas en una prioridad más baja.

Según la forma en que las metas se comparan en función de su grado de importancia, la programación por metas por metas puede clasificarse en dos tipos, con y sin preferencias. Cuando hay preferencias existe una jerarquía de niveles de prioridad en las metas para el decisor. En el caso de sin preferencias en las metas todos los objetivos son de una importancia aproximadamente comparable [21].

En [22] resaltan los aportes de [23] y [24]. [23] señala que el tratamiento dado al problema es bastante completo, preocupándose tanto de los aspectos relacionados con la formulación del modelo, como de lo puramente algorítmico. [24] manifiesta que, desde un punto de vista teórico, se evidencian claros avances, con respecto al trabajo de [23]; demostrando rigurosamente que las técnicas clásicas de programación matemática (lineal, cuadrática, etc.), son casos particulares de la programación por objetivos. Ante estos avances, [25] plantea que el contexto decisional está definido por recursos limitados, en donde el centro decisor, más que optimizar una o varias funciones objetivo, intenta que una serie de metas relevantes se aproximen lo más posible a unos niveles de aspiración fijados de antemano.

Teniendo en cuenta los avances en anteriores publicaciones en [26] se aborda el problema de balance de línea de ensamblaje tipo 2, donde se conoce el número de líneas de estación de trabajo. El objetivo principal no solo es equilibrar el tiempo del ciclo sino también equilibrar la carga de trabajo física de las estaciones simultáneamente. Se desarrolla un modelo de programación de objetivos para el problema.

En [27], se propone el uso del criterio de regularidad y el tiempo de ciclo de Miltenburg como métricas para la integración de los trabajadores y la productividad, respectivamente. Luego definen, modelan y desarrollan una heurística para un problema de equilibrio de línea con estos dos objetivos.
En [28], presenta un enfoque de programación de metas, de números enteros binarios, para lograr un balance de línea mixto restringido por el azar. El método propuesto se ilustra con un ejemplo numérico y una gama diversa de objetivos que un responsable de la toma de decisiones puede enfrentar al equilibrar las líneas.

En este contexto, la programación por metas constituye una metodología que se muestra en un estado de elaboración muy avanzado, siendo objeto de aplicación importante y de interés para la resolución de problemas de balance de línea generales.

\section{III.METODOLOGÍA}

Para determinar la configuración óptima en el problema abordado en este trabajo de tal modo que se minimicen los diferentes objetivos se utilizó la siguiente metodología.

- Basándose en la revisión de la literatura y en el contexto fáctico, se realizó la modelación matemática del problema declarado.

- Se realizó el proceso de caracterización y levantamiento de información en la empresa del sector de farmacéuticos, para poder parametrizar los datos de entrada del modelo.

- Se realiza la implementación del modelo programación por metas en el lenguaje de modelación matemática $L I N G O$ ®.

- Se realiza la validación de los resultados mediante tres escenarios, para garantizar el cumplimiento de las restricciones y refinar los parámetros asociados a las metas.

- Para validar la robustez de la solución obtenida ante la variación de los tiempos de operación, se realizan dos modelos de simulación en ProModel un uno determinístico y otro con variabilidad en los tiempos de operación.

\section{A) Formulación del modelo}

El problema se representa como un modelo de programación con metas no preferentes, es decir todos los objetivos son de una importancia aproximadamente comparables, dado que se manejan penalizaciones expresadas en costos y en tal sentido el modelo propone la optimización de los costos asociados a la minimización del tiempo de ciclo, al número de estaciones y a la agrupación de estas para una operación.

- Para el desarrollo y el análisis del caso se tuvo en cuenta los siguientes supuestos principales.

- Cada estación estará operada por una persona.

- Cada operario trabaja 48 horas a la semana.

- No se tendrá en cuenta las horas extras.

- Todos los turnos tienen 8 horas.

- El máximo número considerado de estaciones permitido es de 17.

- El tiempo de desplazamiento del producto entre estaciones es despreciable. 
- Se pueden agrupar idealmente hasta 4 estaciones por operación, si el modelo así lo considera conveniente. Exceder esta meta es penalizada en la función objetivo y restringida hasta el punto de tener máximo 5 estaciones en paralelo por operación.

\section{B. Notación}

\section{1) Variables}

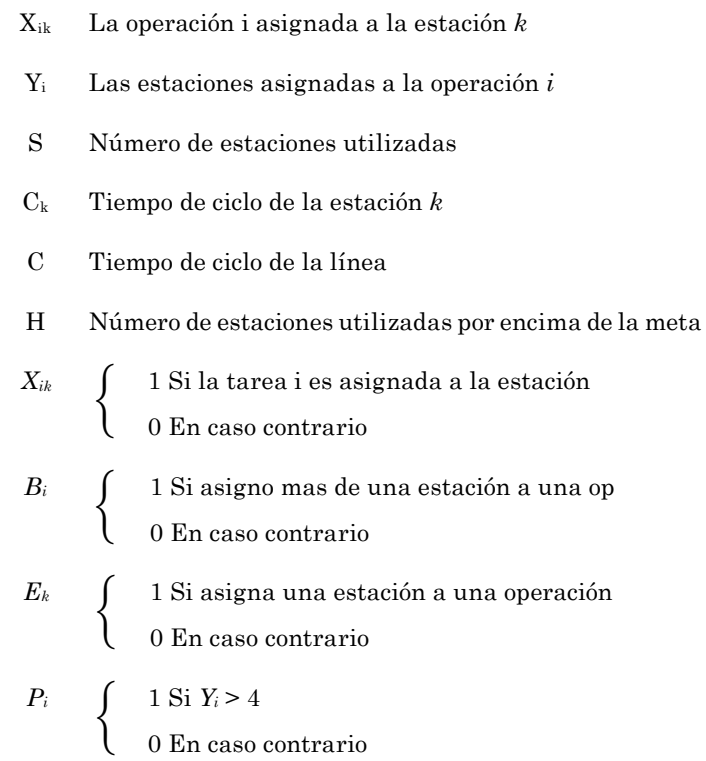

\section{2) Conjuntos}

IP $\{(\mathrm{u}, \mathrm{v})$ : tarea $\mathrm{u}$ debe realizarse primero que la tarea $\mathrm{v}\}$. Conjunto que identifica las restricciones en orden del ensamble

ZD Conjunto de pares de tareas que no pueden ser desarrolladas en una misma estación de trabajo

\section{3) Definición de parámetros}

W El costo de penalizar el exceso de estaciones asignadas a la operación $i$

F El costo de utilizar la línea

L El costo de las estaciones empleadas

G El costo de utilizar una estación por encima de la meta

M Es un valor muy grande

$\mathrm{t}_{\mathrm{i}} \quad$ Tiempo de la operación $i$

\section{4) Formulación matemática}

\section{- Función Objetivo}

Minimizar $\quad(F * C)+(S * L)+(H * G)+W \sum_{i=1}^{N} p_{i}$

\section{- Restricciones}

Tiempo de ciclo

$$
C_{k} \leq C \quad k=1, \ldots, K
$$

Estaciones que se asignan a la operación $i$

$$
Y_{i}=\sum_{k=1}^{K} X_{i k} \quad \mathrm{i}=1, \ldots ., N
$$

Número de estaciones utilizadas

$$
\begin{array}{lr}
\sum_{i=1}^{N} X_{i k} \leq M\left(E_{k}\right) & k=1, \ldots, K \\
1-\sum_{i=1}^{N} X_{i k} \leq M\left(1-E_{k}\right) & k=1, \ldots, K
\end{array}
$$

Condicional para las estaciones que se agrupan para una operación $i$

$$
\begin{aligned}
& Y_{i}-1 \leq M\left(B_{i}\right) \\
& \mathrm{i}=1, \ldots ., N \\
& \sum_{u=1}^{N} X_{u k}-X_{i k}-\left(1-X_{i k}\right) M \leq M\left(1-B_{i}\right) \quad \forall i, k \\
& C_{k}-\frac{t_{i}}{Y_{i}} \leq M\left(2-X_{i k}-B_{i}\right) \quad \begin{array}{l}
i=1, \ldots, N \\
k=1, \ldots, K
\end{array} \\
& C_{k}-\frac{t_{i}}{Y_{i}} \geq-M\left(2-X_{i k}-B_{i}\right) \quad \begin{array}{l}
i=1, \ldots ., N \\
k=1, \ldots, K
\end{array}
\end{aligned}
$$

Condicional para las estaciones que no se agrupan

$$
\begin{aligned}
& 2 o Y_{i} \leq M\left(1-B_{i}\right) \\
& i=1, \ldots, N \\
& \sum_{u=1}^{N} t_{u} X_{u k}-C_{k}-M\left(B_{i}\right) \leq M\left(1-X_{i k}\right) \quad \forall i, k \\
& \sum_{u=1}^{N} t_{u} X_{u k}-C_{k}+M\left(B_{i}\right) \geq-M\left(1-X_{i k}\right) \quad \forall i, k
\end{aligned}
$$

Precedencia

$$
\begin{array}{ll}
X_{v h} \leq \sum_{u=1}^{h} X_{u k} & k=1, \ldots, K ; \\
& (u, v) \in I P
\end{array}
$$

Operaciones separadas

$$
\begin{array}{cl}
X_{u k}+X_{v k} \leq 1 \quad & k=1, \ldots, K ; \\
& (u, v) \in Z_{D}
\end{array}
$$

Número de estaciones utilizadas

$$
\sum_{k=1}^{K} E_{k}=S
$$

Metas propuestas

$$
\begin{aligned}
& Y_{i} \leq 4+P_{i} \quad i=1, \ldots ., N \\
& S \leq 17+H
\end{aligned}
$$


La función objetivo (1) minimiza los costos asociados al tiempo de ciclo, al número de estaciones y a la agrupación de estaciones para una operación; la ecuación (2) garantiza que el tiempo de cada estación no exceda el tiempo de ciclo; la ecuación (3) permite conocer el número de estaciones que se agrupan para una operación i; la ecuación (4) y (5) permiten crear la condición con la ayuda de la ecuación (15) para el registro exacto de las estaciones que se utilizan para el balanceo de la línea: si $X_{i k}=1$ (la operación i se asigno a la estación $\mathrm{k}$ ), obliga a que la variable binaria $E_{k}$ solo puede tomar el valor de 1 , estableciendo el escenario para el conteo.

La restricción (6) es denominada de tipo si entonces, permitiendo activar las restricciones (7), (8) y (9) solo cuando se agrupan más de una estación para una operación i, esto ocurre cuando $Y_{i}>1$ obligando a que la variable binaria tome el valor de 1; la restricción (7) asegura que las estaciones que son asignadas a una operación no sean asignadas a otra operación; las restricciones (8) y (9) verifica que la carga en tiempo de las estaciones agrupadas no exceda el tiempo de ciclo; la restricción (10) presenta la misma conformación del tipo si entonces, permitiendo activar las restricciones (11) y (12) solo cuando se asigna una estación a una operación; las restricciones (11) y (12) aseguran que la carga en tiempo de la estación asignada no exceda el tiempo de ciclo.

La restricción de precedencia (13), representa los requerimientos de secuencia: si $X_{v h}=1$, es decir, si la tarea $\mathrm{v}$ es asignada a la estación $h$ entonces la ecuación debe asumir un valor positivo (para cada predecesor inmediato $v$ de $u$ ), es decir, cada predecesor inmediato u de v debe ser asignado a la estación $k$ tal que $k \leq h$ para que la tarea $u$ sea terminada antes que comience el proceso de la tarea $v$. Si, por lo contrario, $X_{v h}=0$, es decir, si la tarea $\mathrm{v}$ no se asigna a la estación $h$, entonces la ecuación no necesita ser positiva, es decir la predecesora inmediata $u$ de $v$ puede ser o no asignada a la primera estación $h$; la restricción (14) define las operaciones que van separadas, es decir, si $X_{u k}=1$, necesariamente $X_{v k}=0$, garantizando que solo se asigne la operación $u$ a la estación $k$, lo mismo ocurre cuando $X_{u k}=0$, entonces $X_{v k}=1$, certificando que solo se asigne la operación $\mathrm{v}$ a la estación $\mathrm{k}$.

La restricción (15), permite asignar a una variable $S$ el número de veces que se activó la variable binaria logrando contabilizar el número exacto de estaciones utilizadas; las ecuaciones (16) y (17) establecen las metas propuesta por el autor, para el número de estaciones agrupadas y el número máximo de estaciones permitido.

\section{Resultados}

Con el propósito de garantizar un mejor entendimiento de los resultados, primero se presentarán algunos detalles de caso de aplicación, luego se presentarán detalles de la implementación y finalmente la discusión de los resultados

\section{A. Caso de Estudio}

El sector en el que se desarrolla la propuesta es en la industria farmacéutica en esta la elaboración de algunos medicamentos o sub procesos de los mismos, se da mediante un conjunto de actividades que están restringidas en precedencia y con naturaleza discreta, lo que posibilita que se puedan agrupar de varias maneras dentro de un proceso en línea de producción.

La empresa que se emplea como caso de estudio es una empresa farmacéutica de la ciudad de Cali, la planta tiene una capacidad instalada de producción de 1.200.000 unidades semanales, está compuesta por nueve áreas principales que son: manufactura, envase y empaque, control de calidad, bodega, mantenimiento, aseguramiento, estériles y gestión humana.

El modelo de programación por metas para balance de línea se emplea en el área de envase y empaque de píldoras de la compañía. A continuación, se presenta el conjunto operaciones secuenciales para montaje del producto (figura 1).

- El proceso comienza alimentando la máquina, con 25000 Tabletas

- Estas son transportadas por una bandeja vibratoria la cual las conduce a un contador, donde se almacenan hasta llegar al número pedido por orden e inmediatamente se dejan caer para su envasado.

- Seguidamente se encuentra la estación de pesaje, garantizando la cantidad exacta del producto (solo se puede procesar un frasco a la vez).

- El siguiente paso consiste en conducirlos a la banda transportadora donde se le adiciona algodón.

- Luego se ensambla la tapa con el LINER y se le pone la tapa al frasco, e ingresan al horno o túnel para que el LINER se adhiera a la boquilla del frasco.

- Posteriormente se inspecciona si el LINER quedo bien adherido a la boquilla del frasco y luego se coloca en la banda.

- De allí los frascos son transportados por la banda al área de empaque donde se le pone la etiqueta al producto.

- Después se coloca en plegadiza. 
- A continuación, se pone el STICKER a las plegadizas.

- Y el proceso finaliza con la revisión final y la puesta en corrugada del producto.

La obtención de los datos de caso de estudio, se realizó en dos etapas: Análisis de las operaciones, estudio de tiempos y movimientos; y definición de los parámetros para la construcción del modelo de balanceo;

Dentro del desarrollo de este trabajo se empleó el cronometro como instrumento para la medición de la operación realizada por el operario en cada estación; se establecieron 2 ciclos, cada uno compuesto por 10 muestras tomadas en distintos instantes de tiempo.

Luego de haber obtenido los tiempos de cada operación, se adicionó un margen o tolerancia a los datos registrados en el caso de estudio, en el cual se consideran: interrupciones, retrasos, necesidades de los operarios y la disminución del ritmo de trabajo producido por la fatiga inherente a toda labor física y mental. Los resultados obtenidos se observan en la tabla 1 .
Tabla 1. Tiempos de las Operaciones

\begin{tabular}{|c|c|}
\hline Operaciones & $\begin{array}{c}\text { Tiempo } \\
\text { estándar (seg.) }\end{array}$ \\
\hline Pesado (A) & 6.4 \\
\hline Poner algodón (B) & 2.8 \\
\hline Ensamblar tapa con LINER y tapar (C) & 3.6 \\
\hline Inspección tapa con LINER (D) & 5.45 \\
\hline Colocar etiqueta (E) & 15 \\
\hline Poner en plegadiza (F) & 4.69 \\
\hline Poner sticker (G) & 3.1 \\
\hline Revisión y puesta en corrugada (H) & 3.5 \\
\hline
\end{tabular}

Fuente: Autores.

Después de encontrar los tiempos de cada operación se tuvo en cuenta la configuración inicial de la línea de montaje, se observan: los costos, el número de estaciones empleadas, la tasa de producción de la línea y el tiempo empleado en la fabricación de un lote (Tabla 2 y Fig. 1).

Tabla 2. Resumen de costos

\begin{tabular}{|c|c|c|c|c|c|c|c|}
\hline $\begin{array}{c}\text { Costo indirecto } \\
\text { de fabricación (\$) }\end{array}$ & $\begin{array}{c}\text { Costo } \\
\text { estaciones (\$) }\end{array}$ & $\begin{array}{c}\text { Costo } \\
\text { Total (\$) }\end{array}$ & $\begin{array}{c}\text { Und/ } \\
\mathrm{Hr}\end{array}$ & $\begin{array}{c}\text { Terminación } \\
\text { Lote (Horas) }\end{array}$ & $\begin{array}{c}\text { Tiempo de } \\
\text { ciclo (Seg) }\end{array}$ & $\begin{array}{c}\text { Estaciones } \\
\text { Utilizadas }\end{array}$ & $\begin{array}{c}\text { Tiempo } \\
\text { Ocioso (\%) }\end{array}$ \\
\hline 682,667 & 366,182 & $1,048,849$ & 563 & 13.65 & 6.4 & 10 & 30 \\
\hline
\end{tabular}

Fuente: Autores.

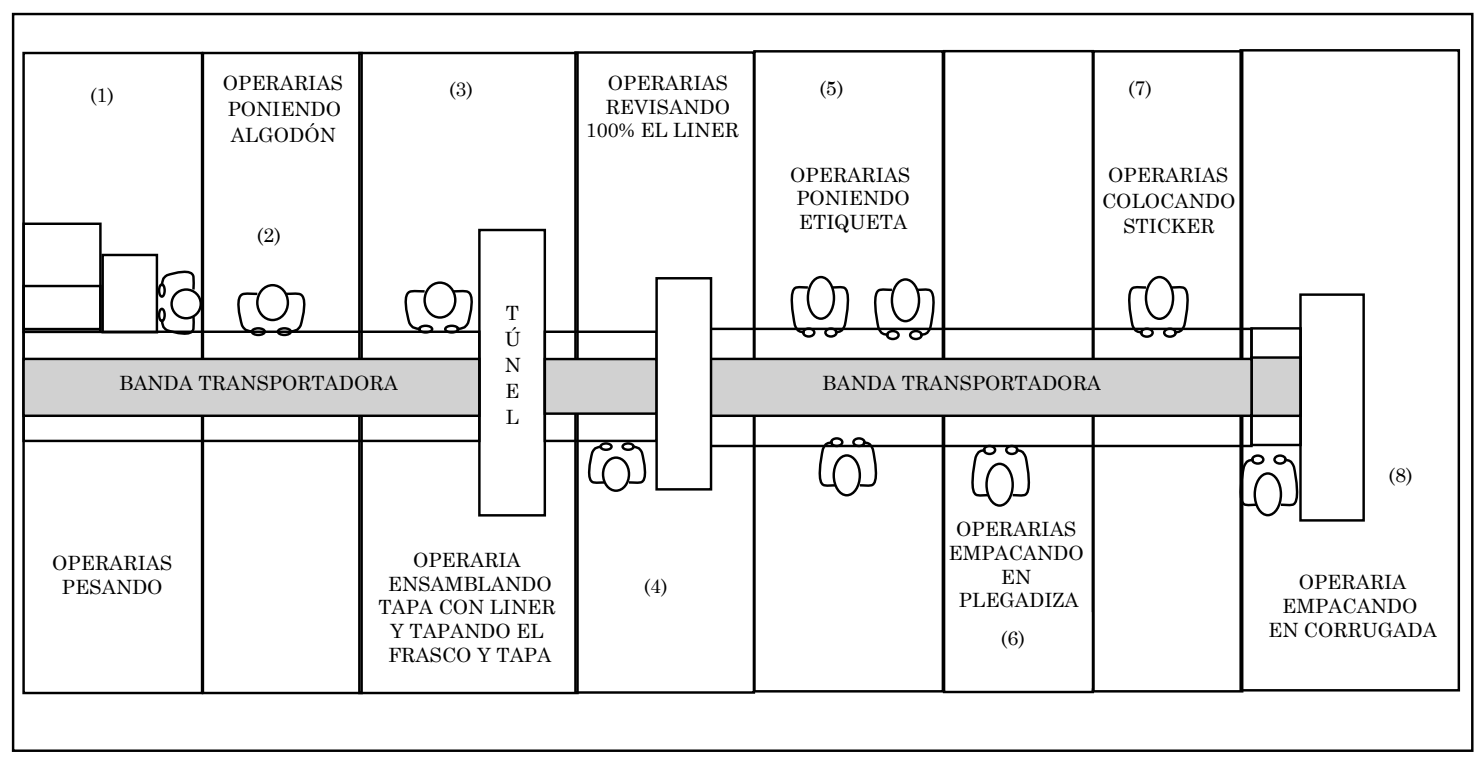

Fig. 1. Distribución Inicial. Fuente: Autores. 
Para la aplicación del modelo de programación por metas también se tuvo en cuenta los siguientes datos: el máximo número considerado de estaciones permitido es de 17, el tiempo de desplazamiento del producto entre estaciones es despreciable, el costo de utilizar la línea por hora es de \$50.000. Además de contar con dos turnos de 8 horas, el lote de producción está compuesto por 7.680 unidades, el salario establecido fue el mínimo legal vigente, se tuvo en cuenta un valor por hora.

Por otro lado se tuvieron en cuenta otros parámetros como el Costo del uso de una estación (L); [29] suponen que en la operación de una estación $k$, se causa una tasa de salario por unidad de tiempo, que es igual a la remuneración máxima de todas las tareas que se asignan a esa estación. También se tuvo en cuenta el Costo indirecto de la fabricación de un lote (F) y Costo de utilizar más de 17 estaciones $(\mathrm{H})$.

\section{B. Implementación del modelo}

Con la información obtenida del caso de estudio, se implementó el modelo de programación por metas en el lenguaje de modelación matemática Lingo ${ }^{\circledR}$; Durante la implementación se observó el comportamiento del modelo propuesto, obteniendo como resultado un máximo de 207 variables, 11 no lineales y 161 enteras. Se desarrollaron tres escenarios de validación de resultados.
- Para el primer escenario, sólo se minimizó el costo asociado al tiempo de ciclo, con un número máximo de 17 estaciones y 8 operaciones, el número de variables es de 207, no lineales 11 y enteras 161 .

- Para el segundo escenario, a diferencia del primero, sólo se cambió la función objetivo y se minimizó el costo asociado al número de estaciones, generándose un total de 205 variables, 11 no lineales y 143 enteras.

- Para el tercer escenario se corrió el modelo propuesto, dando como resultado un máximo de 207 variables, 11 no lineales y 161 enteras.

A través de esta implementación, se validó el cumplimiento de las restricciones planteadas, la precedencia de las operaciones y el buen funcionamiento del modelo mediante las soluciones óptimas obtenidas.

\section{Análisis de Resultados}

En el primer escenario, se minimizó solo el tiempo de ciclo asociado a un costo, con el fin de observar el comportamiento del modelo; generando como resultado el manejo de todas las estaciones y la reducción al máximo la función objetivo, Aunque no se incluyó la minimización del costo asociado a las estaciones; en la función objetivo, se contabilizó el número de estaciones utilizadas con el fin de obtener un costo total y establecer un argumento válido, los resultados son: costo total de $\$ 652.588,1.125$ unidades por hora y un tiempo de ciclo de 3,2 segundos, los demás resultados y la distribución por operación, tal como se observa en la tabla 3 , tabla 4 y Fig. 2.

Tabla 3. Resumen del PRimer escenario.

\begin{tabular}{|c|c|c|c|c|c|c|}
\hline $\begin{array}{c}\text { Costo indirecto de } \\
\text { fabricación (\$) }\end{array}$ & $\begin{array}{c}\text { Costo } \\
\text { estaciones }(\$)\end{array}$ & $\begin{array}{c}\text { Costo } \\
\text { Total }(\$)\end{array}$ & Und/Hr & $\begin{array}{c}\text { Terminación } \\
\text { Lote (Horas) }\end{array}$ & $\begin{array}{c}\text { Tiempo de } \\
\text { ciclo (seg) }\end{array}$ & $\begin{array}{c}\text { Estaciones } \\
\text { Utilizadas }\end{array}$ \\
\hline 341.333 & 311.255 & 652.588 & 1.125 & 6,83 & 3,2 & 17 \\
\hline
\end{tabular}

Fuente: Autores.

Tabla 4. Configuración primer escenario, Agrupación de estaciones Por operación CON SUS RESPECtivos tiempos - PRimer escenario

\begin{tabular}{|c|c|c|c|c|c|c|c|c|c|c|c|c|c|c|c|c|c|c|}
\hline$t(s)$ & & EST1 & EST2 & EST3 & EST4 & EST5 & EST6 & EST7 & EST8 & EST9 & EST10 & EST11 & EST12 & EST13 & EST14 & EST15 & EST16 & EST17 \\
\hline 6,4 & OP1 & 1 & 1 & & & & & & & & & & & & & & & \\
\hline 2,8 & OP2 & & & 1 & & & & & & & & & & & & & & \\
\hline 3,6 & OP3 & & & & 1 & & & & & & & & & & & 1 & & \\
\hline 5,45 & OP4 & & & & & 1 & 1 & & & & & & & & & & & \\
\hline 15 & OP5 & & & & & & & 1 & 1 & 1 & 1 & 1 & & & & & & \\
\hline 4,69 & OP6 & & & & & & & & & & & & 1 & 1 & & & & \\
\hline 3,1 & OP7 & & & & & & & & & & & & & & 1 & & & \\
\hline 3,5 & OP8 & & & & & & & & & & & & & & & & 1 & 1 \\
\hline & & 3,2 & 3,2 & 2,8 & 1,8 & 2,73 & 2,73 & 3 & 3 & 3 & 3 & 3 & 2,35 & 2,35 & 3,1 & 1,8 & 1,75 & 1,75 \\
\hline
\end{tabular}

Fuente: Autores. 


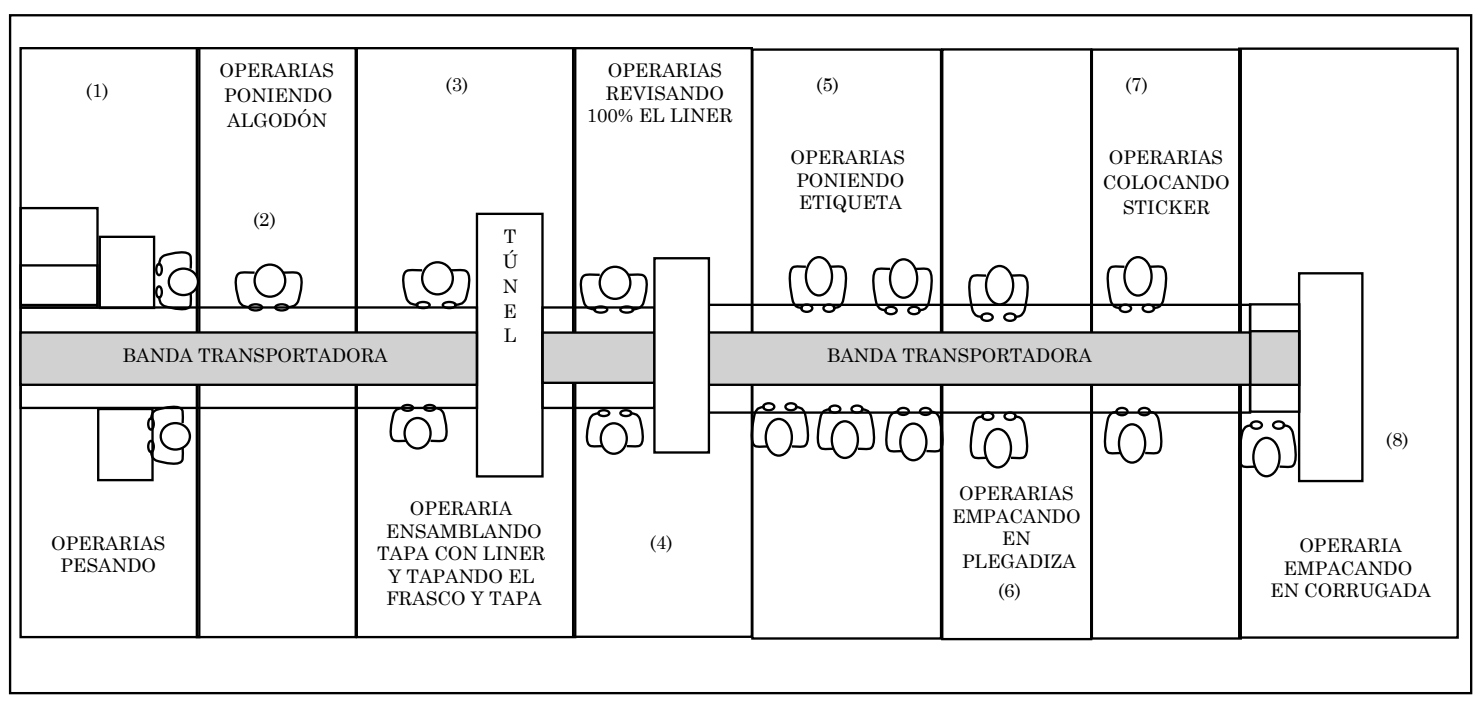

Fig. 2. Distribución primer escenario.

Fuente: Autores.

Tabla 5. Resumen SEgundo escenario

\begin{tabular}{|c|c|c|c|c|c|c|c|}
\hline $\begin{array}{c}\text { Costo indirecto de } \\
\text { fabricación (\$) }\end{array}$ & $\begin{array}{c}\text { Costo } \\
\text { estaciones (\$) }\end{array}$ & $\begin{array}{c}\text { Costo } \\
\text { Total }(\$)\end{array}$ & Und/Hr & $\begin{array}{c}\text { Terminación } \\
\text { Lote (Horas) }\end{array}$ & $\begin{array}{c}\text { Tiempo de } \\
\text { ciclo (seg) }\end{array}$ & $\begin{array}{c}\text { Estaciones } \\
\text { Utilizadas }\end{array}$ & $\begin{array}{c}\text { Tiempo } \\
\text { Ocioso (\%) }\end{array}$ \\
\hline 1.600 .000 & 686.592 & 2.286 .592 & 240 & 32 & 15 & 8 & 62,9 \\
\hline
\end{tabular}

Fuente: Autores.

Tabla 6. Configuración primer escenario, Agrupación de estaciones Por operación CON Sus RespeCtivos tiempos - Segundo escenario

\begin{tabular}{|c|c|c|c|c|c|c|c|c|c|c|c|c|c|c|c|c|c|c|}
\hline $\mathrm{t}(\mathrm{s})$ & & EST1 & EST2 & EST3 & EST4 & EST5 & EST6 & EST7 & EST8 & EST9 & EST10 & EST11 & EST12 & EST13 & EST14 & EST15 & EST16 & EST17 \\
\hline 6,4 & OP1 & 1 & & & & & & & & & & & & & & & & \\
\hline 2,8 & OP2 & & & 1 & & & & & & & & & & & & & & \\
\hline 3,6 & OP3 & & & & 1 & & & & & & & & & & & & & \\
\hline 5,45 & OP4 & & & & & 1 & & & & & & & & & & & & \\
\hline 15 & OP5 & & & & & & & 1 & & & & & & & & & & \\
\hline 4,69 & OP6 & & & & & & & & & & & & 1 & & & & & \\
\hline 3,1 & OP7 & & & & & & & & & & & & & & 1 & & & \\
\hline 3,5 & OP8 & & & & & & & & & & & & & & & & 1 & \\
\hline & & 6,4 & 0 & 2,8 & 3,6 & 5,45 & 0 & 15 & 0 & 0 & 0 & 0 & 4,69 & 0 & 3,1 & 0 & 3,5 & 0 \\
\hline
\end{tabular}

Fuente: Autores.

Se estableció el mismo escenario, pero esta vez solo minimizando el número de estaciones asociadas a un costo sin metas. Con el propósito de ver el desempeño del modelo, se decidió, como en el caso anterior, costear el tiempo de ciclo empleado en la optimización, alcanzando el siguiente resultado: costo total de $\$ 2.286 .592,240$ unidades por hora y un tiempo de ciclo de 15 segundos. Para efecto de ilustrar los resultados y la distribución de las estaciones, se presentan a continuación en la tabla 5, tabla 6 y Fig. 3.
$\mathrm{Al}$ analizar estos dos planteamientos, se encontró un aumento en el costo total en más de un $350 \%$, esto se debe a que la tasa de producción del escenario 2 es muy baja (tabla 5), lo que ocasiona un mayor uso de las estaciones de la línea, incurriendo en un costo más alto. Por otro lado, al reducir el tiempo de ciclo (tabla 3) se aumenta la productividad utilizando todas las estaciones disponibles, generando la ocupación máxima de la línea; Este escenario es interesante ya que se podría ver como la mejor configuración que se puede obtener, sin embargó se presentan cos- 


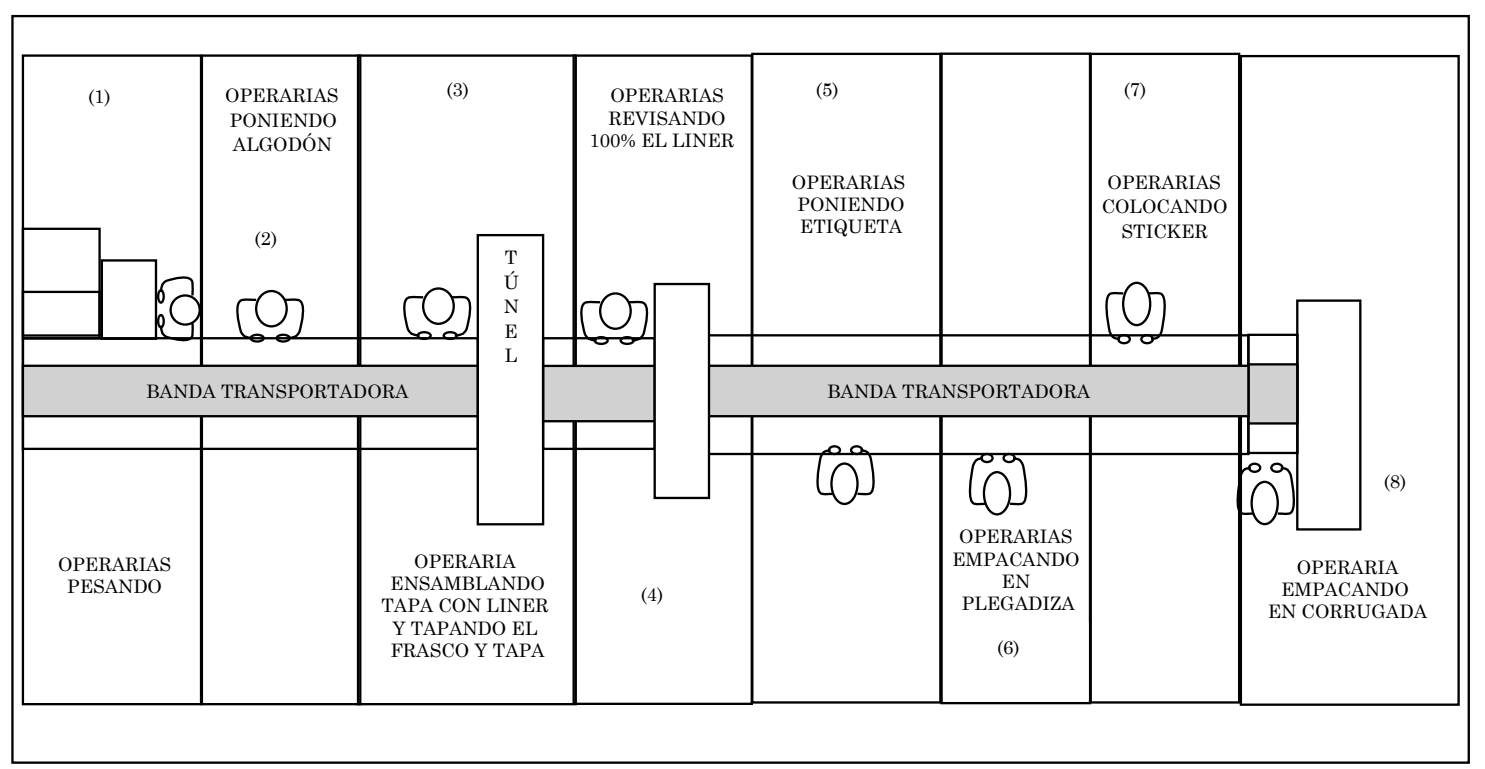

Fig. 3. Distribución Segundo escenario. Fuente: Autores.

tos que no se contabilizan como el tiempo ocioso, el exceso de estaciones agrupadas para una operación y el número máximo permitido de estaciones.

De acuerdo con los hallazgos, cabe resaltar que en la comparación de los anteriores escenarios, se estableció como función objetivo, la minimización de los costos asociados: al tiempo de ciclo, al número de estaciones utilizadas y a la agrupación de estas con las metas propuestas, obteniendo como resultado un costo total de $\$ 700.384,960$ unidades por hora y un tiempo de ciclo de 3,75 segundos. Para otros resultados y la distribución de las estaciones se presentan a continuación en la tabla 7, tabla 8 y Fig. 4.

Tabla 7. Resumen tercer escenario

\begin{tabular}{|c|c|c|c|c|c|c|c|}
\hline $\begin{array}{l}\text { Costo indirecto de } \\
\text { fabricación }(\$)\end{array}$ & $\begin{array}{c}\text { Costo } \\
\text { estaciones }(\$)\end{array}$ & $\begin{array}{c}\text { Costo } \\
\text { Total }(\$)\end{array}$ & Und/Hr & $\begin{array}{l}\text { Terminación } \\
\text { Lote }(\mathrm{Hr})\end{array}$ & $\begin{array}{l}\text { Tiempo de } \\
\text { ciclo (s) }\end{array}$ & $\begin{array}{l}\text { Estaciones } \\
\text { Utilizadas }\end{array}$ & $\begin{array}{c}\text { Tiempo } \\
\text { Ocioso (\%) }\end{array}$ \\
\hline 400.000 & 300.384 & 700.384 & 960 & 8,00 & 3,75 & 14 & 15 \\
\hline
\end{tabular}

Fuente: Autores.

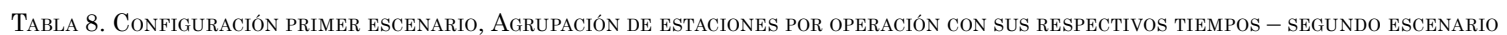

\begin{tabular}{|c|c|c|c|c|c|c|c|c|c|c|c|c|c|c|c|c|c|c|}
\hline$t(s)$ & & EST1 & EST2 & EST3 & EST4 & EST5 & EST6 & EST7 & EST8 & EST9 & EST10 & EST11 & EST12 & EST13 & EST14 & EST15 & EST16 & EST17 \\
\hline 6,4 & OP1 & 1 & 1 & & & & & & & & & & & & & & & \\
\hline 2,8 & OP2 & & & 1 & & & & & & & & & & & & & & \\
\hline 3,6 & OP3 & & & & 1 & & & & & & & & & & & & & \\
\hline 5,45 & OP4 & & & & & 1 & 1 & & & & & & & & & & & \\
\hline 15 & OP5 & & & & & & & 1 & 1 & 1 & 1 & & & & & & & \\
\hline 4,69 & OP6 & & & & & & & & & & & & 1 & 1 & & & & \\
\hline 3,1 & 0P7 & & & & & & & & & & & & & & 1 & & & \\
\hline 3,5 & 0P8 & & & & & & & & & & & & & & & & 1 & \\
\hline & & 3,2 & 3,2 & 2,8 & 3,6 & 2,73 & 2,73 & 3,75 & 3,75 & 3,75 & 3,75 & 0 & 2,35 & 2,35 & 3,1 & 0 & 3,5 & 0 \\
\hline
\end{tabular}

Fuente: Autores. 


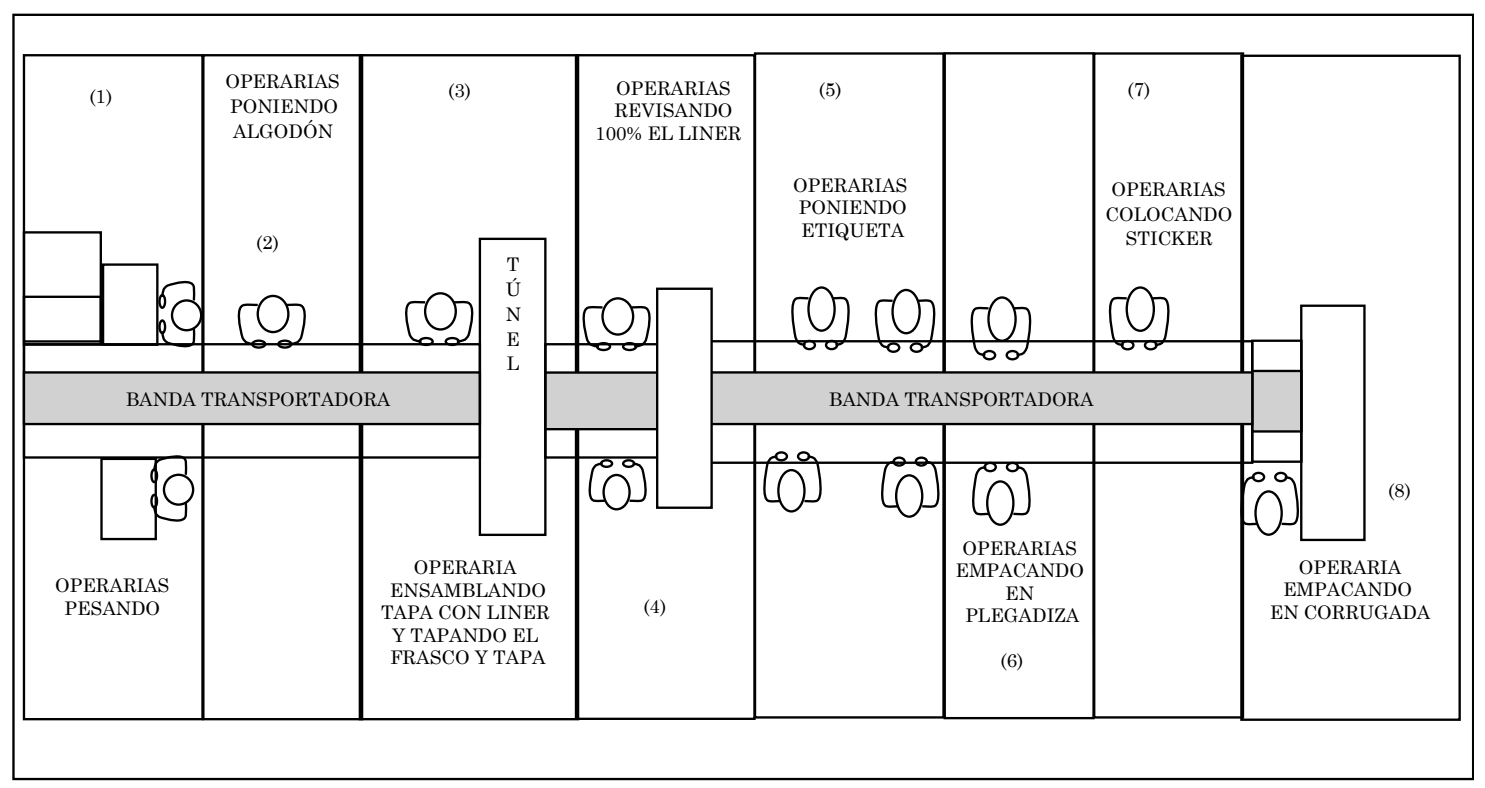

Fig. 4. Distribución Tercer escenario. Fuente: Autores.

Tal como se puede observar entre la configuración inicial y la configuración final (tabla 2 y tabla 7), se presenta una disminución importante del tiempo ciclo y del tiempo ocioso, sin embargo se incrementaron en un $40 \%$ el número de estaciones, esto se ve compensado con el aumento en la utilización racional de los recursos, el número de unidades producidas y el tiempo de utilización de las estaciones, logrando sacar un lote en un turno de 8 horas, mientras que antes se lograba aproximadamente en 14. De otra parte, uno de los beneficios obtenidos con esta propuesta es lograr despejar la línea más rápido, permitiendo que se pueda entrar a envasar y empacar otro producto, con lo que se logra reducir el costo total en un $33.22 \%$, consiguiendo que la empresa sea más eficiente en su proceso de manufactura.

Con el fin de profundizar en el análisis de los resultados de la solución obtenida en el sistema y con el ánimo de analizar su robustez frente a variaciones en el tiempo de operación, se simuló la solución de balance de línea obtenida en el modelo por metas en dos modelos de simulación empleando el software ProModel.

El primer modelo de simulación es determinístico en el cual se trabajó con el tiempo estándar de cada una de las operaciones obtenida en el estudio de tiempos, En el segundo modelo de simulación se considera la variabilidad en los tiempos de operación, en este caso se identificó la máxima variabilidad en los tipos estándar de las operaciones, la cual fue del $10 \%$ y se utilizó la distribución normal, tomando como media el tiempo estándar de cada operación.
Luego de realizar la simulación de la configuración inicial, presentada en la empresa, y a partir del análisis de los resultados (Fig. 5), se encontró que la línea de montaje manifiesta un cuello de botella en la estación de pesado con un 100\% de utilización, ocasionando un $30 \%$ de inactividad o tiempo ocioso lo que conlleva a una tasa de producción más baja causando un aumento en el tiempo de terminación del lote y del costo total de la línea (Ver tabla 2).

Con el objetivo de mejorar este escenario, el modelo propuesto distribuyó la carga de la estación cuello de botella, en varias estaciones considerando unos supuestos y condiciones del entorno que permitieron mejorar la tasa de producción en un $43 \%$, con respecto al escenario inicial, también se logró bajar el porcentaje de tiempo ocioso de un $30 \%$ a un $15 \%$. Conjuntamente se disminuye el tiempo de proceso de un lote en un $40 \%$, generando un aumento en la eficiencia cercano al $85 \%$.

Adicionalmente, se presenta el análisis del modelo de simulación determinístico vs el modelo simulación con variabilidad obteniendo los siguientes datos. Para el modelo determinístico se encontró un tiempo de ciclo de 3.75 segundos, utilizando 14 estaciones y produciendo 960 unidades por ahora; mientras que, en el modelo de simulación con variabilidad, el tiempo de ciclo arrojado es de 3.72 segundos, empleando 14 estaciones y con una tasa de producción de 968 unidades por hora (Tabla 9). En la simulación, se puede observar la robustez de la solución debido a que no presenta variaciones significativas con respecto al modelo determinístico, generando un resultado satisfactorio que da cumplimiento a los objetivos planteados en este trabajo. 


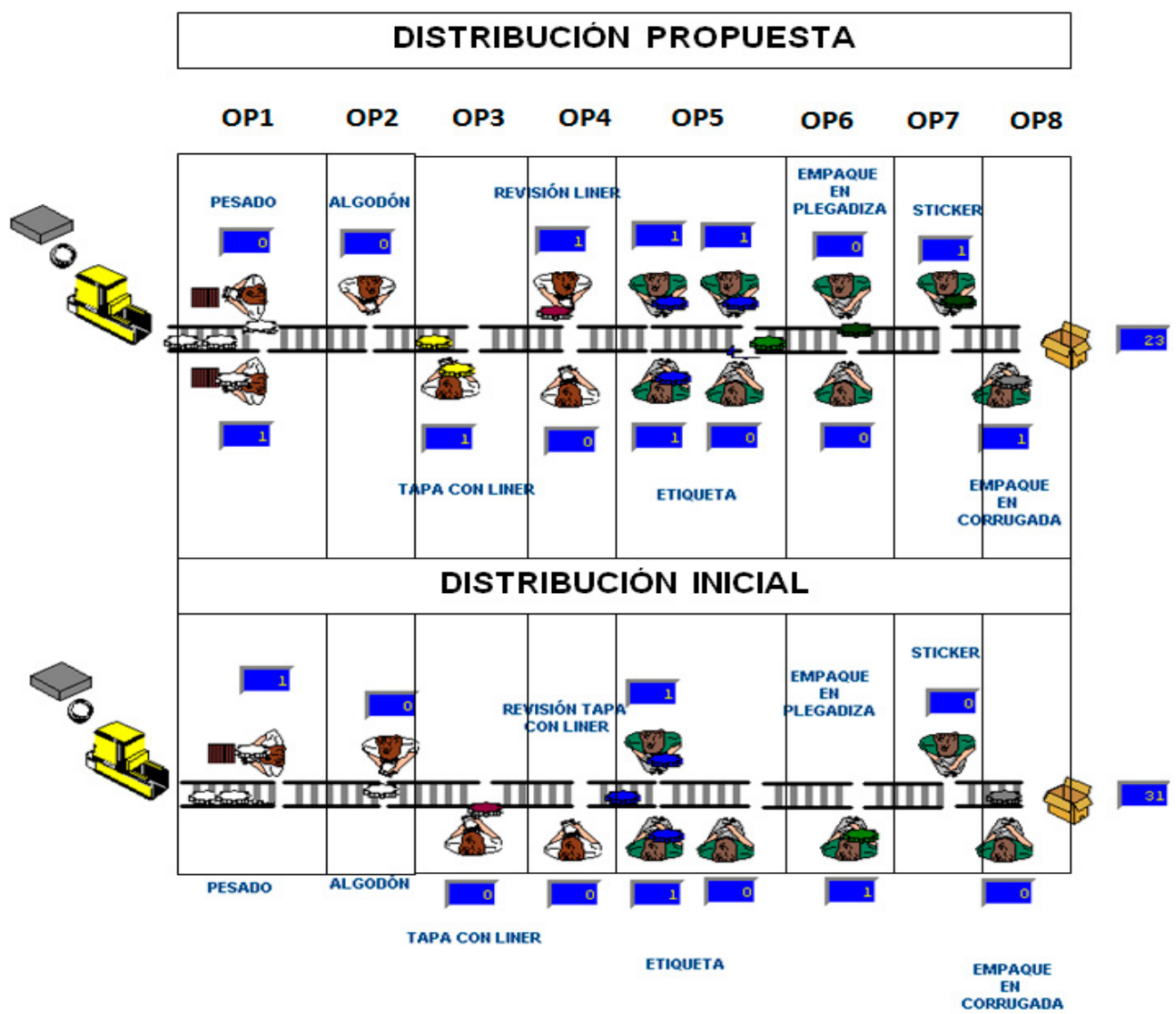

Figura 5. Distribución propuesta vs la distribución inicial Fuente: Autores.

Tabla 9. Resultados de los modelos

\begin{tabular}{|c|c|c|c|c|c|}
\hline & $\begin{array}{c}\text { Tiempo de } \\
\text { ciclo }\end{array}$ & $\begin{array}{c}\text { Número de } \\
\text { estaciones }\end{array}$ & $\begin{array}{c}\text { Tiempo de finalización } \\
\text { (Horas) }\end{array}$ & $\begin{array}{c}\text { Tiempo ocioso } \\
\text { (\%) }\end{array}$ & $\begin{array}{c}\text { Tasa de producción } \\
\text { (Und/Hora) }\end{array}$ \\
\hline Modelo optimizado con Lingo & 3.75 & 14 & 8 & 15 & 960 \\
\hline $\begin{array}{c}\text { Modelo de simulación con } \\
\text { ProModel }\end{array}$ & 3.72 & 14 & 7.9 & 15 & 968 \\
\hline
\end{tabular}

Fuente: Autores.

\section{Conclusiones}

Es importante establecer un balance, entre estas dos variables, con el fin de mejorar la eficiencia y la optimización de los costos, obteniendo una mayor flexibilización de los procesos de producción, para la elaboración de diferentes productos, que den respuesta a las demandas actuales y futuras de la organización.

La simulación, es una herramienta que permite ilustrar la complejidad de las operaciones del sistema de producción que requieren como en nuestro caso de una modelación más ajustada a la realidad, para efecto de comprender con mayor profundidad el comportamiento del proceso y poder evaluar varias estrategias.
Al incrementar los valores de los parámetros en un $50 \%, 100 \%, 150 \%$ y $200 \%$, se encontró que los resultados arrojados por el modelo no sufrieron variaciones con respecto al primer incremento, sin embargo, el modelo si sufre modificaciones con respecto al parámetro $\mathrm{F}$ cuando se incrementa en un $100 \%$ ya que se modifica la configuración obtenida.

En el análisis de los trabajos publicados, se puede observar que la mayoría trata problemas de balanceo de línea mono-objetivo, debido a que se quiere determinar o elegir, entre todas las alternativas posibles, aquella que posee un mayor grado de deseabilidad para el decisor, es decir la solución óptima; Encontrando que pocas veces en la vida real es posible evaluar una situación y decidir con base a un solo criterio. 
La programación por metas, se muestra como un elemento de apoyo, al combinar la lógica tradicional de la optimización, y el deseo de los centros decisores de satisfacer diferentes metas, convirtiéndose en un instrumento analítico de gran potencialidad, dada su importancia en ambientes de incertidumbre.

Considerar el concepto de estaciones en paralelo, permite lograr que las operaciones que presentan tiempos mayores al tiempo de ciclo, se le asigne más de una estación, con el fin de conseguir el equilibrio deseado en el flujo de la línea.

Se propone como investigación futura, abordar el problema de balance de línea propuesto, mediante técnicas de optimización multi-objetivo de tal modo que se pueda construir un frente de Pareto con múltiples soluciones que represente puntos óptimos al problema propuesto.

Los aspectos sociales juegan cada vez más un papel importante en el balance de línea, en tal sentido se propone como investigación futura, introducir al modelo una función de desempeño orientada al bienestar social.

\section{FinANCIAMIENTO}

Artículo de investigación derivado del proyecto titulado "Balance de línea en sistemas de fabricación con tiempos de proceso dominantes". Financiado por la Universidad del Valle. Cali, Colombia. Fecha de inicio: 08/02/2015. Fecha de finalización: 02/07/2017

\section{REFERENCIAS}

[1] N. Boysen, M. Fliedner and A. Scholl, "A classification of assembly line balancing problems," Eur. J. Oper. Res., vol. 183 , no. 2 , pp. $674-693$, Dec. 2007 , doi: https://doi. org/10.1016/j.ejor.2006.10.010

[2] J. Pereira and E. Álvarez-Miranda, "An exact approach for the robust assembly line balancing problem," Omega, vol. 78, pp. 85-98, Jul. 2018, doi: https://doi. org/10.1016/j.omega.2017.08.020

[3] I. Baybars, "A Survey of Exact Algorithms for the Simple Assembly Line Balancing Problem," Manage. Sci., vol. 32, no. 8, pp. 909-932, Aug. 1986, doi: https://doi. org $/ 10.1287 / \mathrm{mnsc} .32 .8 .909$

[4] E. Erel, "A study on production lines with discrete batch WIP inventory transfer," Int. J. Prod. Econ., vol. 55, no. 1, pp. 71-85, Jun. 1998, doi: https://doi.org/10.1016/ S0925-5273(98)00042-5

[5] E. Erel, "Effect of discrete batch WIP transfer on the efficiency of production lines," Int. J. Prod. Res., vol. 31, no. 8, pp. 1827-1838, Aug. 1993, doi: https://doi. org/10.1080/00207549308956825.

[6] P. J. Egbelu and N. Roy, "Material flow control in AGV/ unit load based production lines," Int. J. Prod. Res., vol. 26 , no. 1 , pp. $81-94$, Jan. 1988 , doi: https://doi. org/10.1080/00207548808947842

[7] P. J. Egbelu, "Batch production time in a multi-stage system with material-handling consideration," Int. J. Prod. Res., vol. 29, no. 4, pp. 695-712, Apr. 1991, doi: https://doi.org/10.1080/00207549108930096

[8] P. J. Egbelu, "Machining and material flow system design for minimum cost production," Int. J. Prod. Res., vol. 28 , no. 2 , pp. $353-368$, Feb. 1990, doi: https://doi. org/10.1080/00207549008942716
[9] R. G. Qiu, "Virtual production line based WIP control for semiconductor manufacturing systems," Int. J. Prod. Econ., vol. 95, no. 2, pp. 165-178, Feb. 2005, doi: https:// doi.org/10.1016/j.ijpe.2003.12.004

[10] W. Choi and Y. Lee, "A dynamic part-feeding system for an automotive assembly line," Comput. Ind. Eng., vol. 43, no. 1-2, pp. 123-134, Jul. 2002, doi: https://doi. org/10.1016/S0360-8352(02)00071-2

[11] A. Scholl and R. Klein, "SALOME: A Bidirectional Branch-and-Bound Procedure for Assembly Line Balancing," INFORMS J. Comput., vol. 9, no. 4, pp. 319-334, Nov. 1997, doi: https://doi.org/10.1287/ijoc.9.4.319

[12] P.R.McMullen and P. Tarasewich, "Multi-objective assembly line balancing via a modified ant colony optimization technique," Int. J. Prod. Res., vol. 44, no. 1, pp. 27-42, Jan. 2006, doi: https://doi.org/10.1080/00207540500216748

[13] A. Scholl and C. Becker, "State-of-the-art exact and heuristic solution procedures for simple assembly line balancing," Eur. J. Oper. Res., vol. 168, no. 3, pp. 666-693, Mar. 2006, doi: https://doi.org/10.1016/j.ejor.2004.07.022

[14] Y. Bukchin and T. Raviv, "Constraint programming for solving various assembly line balancing problems," Omega, vol. 78, pp. 57-68, Jul. 2018, doi: https://doi. org/10.1016/j.omega.2017.06.008

[15] J. Pereira and E. Álvarez-Miranda, "An exact approach for the robust assembly line balancing problem," Omega, vol. 78, pp. 85-98, Jul. 2018, doi: https://doi.org/10.1016/j. omega.2017.08.020

[16] J. R. Coronado-Hernandez and H. Ospina-Mateus, "Incorporación de Riesgos Ergonómicos en el Balanceo de Líneas de Ensamble en U," WPOM - Work. Pap. Oper. Manag., vol. 4, no. 2, pp. 29-43, Sept. 2013, doi: http:// dx.doi.org/10.4995/wpom.v4i2.1164

[17] E. H. Bowman, "Assembly-Line Balancing by Linear Programming," Oper. Res., vol. 8, no. 3, pp. 385-389, Jun. 1960. doi: http://dx.doi.org/10.1287/opre.8.3.385

[18] C. N. Klahr, "Multiple Objectives in Mathematical Programming," Oper. Res., vol. 6, no. 6, pp. 849-855, Nov. 1958. Available: https://doi.org/10.1287/opre.6.6.849

[19] A. Charnes and W. W. Cooper, Management models and industrial applications of linear programming, vol. 1, N.J., USA: John Wiley \& Sons, 1961.

[20] Y. Ijiri, Management goals and accounting for control, Haarlem, Netherlands: North Holland Pub. Co., 1965.

[21] F. S. Hillier and G. J. Lieberman, Investigación de operaciones, 10th ed., Madrid, España: McGraw-Hill Interamericana S.L., 2016.

[22] C. Romero, "El enfoque multiobjetivo en los modelos matemáticos de planificación de cultivos," Rev. Econ. Polit., vol. 89, pp. 179-204, Sept. 1981.

[23] S. M. Lee, Goal Programming for Decision Analysis, Philadelphia, USA: Auerbach Pub., 1972.

[24] J. P. Ignizio, Goal programming and extensions, Toronto, Canada: Lexington Books, 1976.

[25] C. Romero, Análisis de las decisiones multicriterio, vol. 14, Madrid, España: Isdefe, 1996.

[26] Ö. Polat, Ö. Mutlu and E. Özgörmüş, "A Goal Programming Model For Assembly Line Balancing Problem Type 2 Under Workload Constraint." in The 2015 Northeast Decision Sciences Conf., Cambridge, MA, March 2015.

[27] M. C. O. Moreira, R. Pastor, A. M. Costa and C. Miralles, "The multi-objective assembly line worker integration and balancing problem of type-2," Comput. Oper. Res., vol. 82, pp. 114-125, Jun. 2017, doi: https://doi.org/10.1016/j. cor.2017.01.003

[28] A. J. R. Smith and A. Bhandari, "Multiple criteria approach to the stochastic assembly line balancing problem," Proc. 33rd Int. Matador Conf., 2000, pp. 61-66.

[29] O. Rosenberg and H. Ziegler, "A comparison of heuristic algorithms for cost-oriented assembly line balancing," ZOR Zeitschrift fü Oper. Res. Methods Model. Oper. Res., vol. 36 , no. 6, pp. 477-495, Nov. 1992. Available: https:// link.springer.com/article/10.1007/BF01416240 
Juan Pablo Orejuela Cabrera: Recibió su título de Magister en ingeniería con énfasis en ingeniería industrial de la Universidad del Valle, Cali, Colombia, en el año 2008. Y su grado de Ingeniero industrial de la Universidad del Valle, Cali, Colombia en el año 2001. Es Profesor Tiempo completo e integrante del grupo de investigación de Producción y logística de la Universidad del Valle, Cali, Colombia. https://orcid. org/0000-0003-2187-0630

Andrés Flórez González: Recibió su título de Ingeniero industrial de la Universidad del Valle, Cali, Colombia. Sus intereses de investigación incluyen Logística, Producción, Optimización de Cadenas de Abastecimiento. https://orcid.org/0000-0002-78575440 